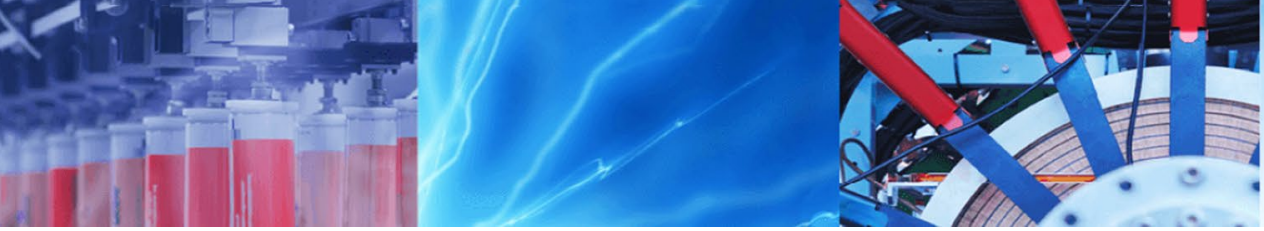

Research Article

\title{
Enzyme-free colorimetric sensing of glucose using L-cysteine functionalized silver nanoparticles
}

\author{
Sumaira Adnan ${ }^{1} \cdot$ Nazar Hussain Kalwar ${ }^{2,3,4} \cdot$ Malik Waseem Abbas $^{1} \cdot$ Razium Ali Soomro $^{1,4,5} \cdot$ Mumtaz Ali Saand $^{6}$. \\ Fazli Rabbi Awan ${ }^{1} \cdot$ Ahmet Avci $^{3}$. Erol Pehlivan ${ }^{7} \cdot$ Sadia Bajwa $^{1}$
}

(c) Springer Nature Switzerland AG 2019

\begin{abstract}
The study demonstrates an efficient, simple and on-site viable approach for sensitive determination of blood glucose using colorimetric sensing approach. The devised colorimetric sensor works based on cysteine functionalized silver nanoparticles. The as-synthesized Ag NPs were elaborately characterized using advanced analytical techniques such as ultra-violet visible spectroscopy, Fourier transform infrared spectroscopy, transmission electron microscopy, atomic force microscopy and X-ray diffraction. The detection of glucose was carried under ambient air conditions where change in the optical characteristics of Ag NPs and subsequent interaction between glucose and functional moiety (i.e. surface bind cysteine) of Ag NPs was considered as the signal response. This interaction led to direct detection of glucose in the concentration range between 0.01 to $0.17 \mu \mathrm{M}$ with limit of detection up to $1 \times 10^{-4} \mu \mathrm{M}$. It is worthwhile mentioning that success of the assay lies in application of the developed colorimetric sensor in real blood glucose measurements, which also proved its capability for field based analysis. In addition its simple design, low cost, and more reliability signifies the usefulness of colorimetric sensor and it can be extended monitor other biologically important molecules.
\end{abstract}

Keywords Glucose · Enzyme free · Colorimetric probes · Functionalized Ag NPs

\section{Introduction}

As diabetes mellitus have become pressing health problem around the globe when dealing with glucose metabolism disorders. This chronic disease, associated with insulin deficiency/resistance may lead to heart or kidney failure, high blood pressure, nerve damage and blindness due to continuous variation in blood glucose [1]. Thus, research on portable devices and chemical assays which can allow fast, sensitive and reliable monitoring of blood glucose level is an area of scientific research gaining significant interest of the people. Conventional approaches which include high-performance liquid chromatography (HPLC)
[2], fluorescence spectrometry and chemiluminescence methods [2], despite being sensitive, fail to offer advantages like fast analysis, affordability, portability and ease of analysis. In contrast, colorimetric assay based on the use of nano-sized noble metal nanoparticles such as gold or silver have proven efficient, cheap and capable for onsite monitoring or screening of biologically important molecules. Among various metals available, silver and gold are most extensively used for the development of colorimetric probes based on their excellent surface plasmon resonance (SPR) characteristics. However, the use of such noble metals for probing biologically important molecules for example glucose is normally associated

Nazar Hussain Kalwar, nazarkalwar@gmail.com | ${ }^{1}$ National Institute for Biotechnology and Genetic Engineering (NIBGE), Faisalabad 38000, Pakistan. ${ }^{2}$ Institute of Chemistry, Shah Abdul Latif University, Khairpur 66020, Pakistan. ${ }^{3}$ Department of Mechanical Engineering, Faculty of Engineering, University of Selcuk, Campus, 42079 Konya, Turkey. ${ }^{4}$ Interface Analysis Centre, School of Physics, University of Bristol, Bristol BS8 1TL, UK. ${ }^{5}$ National Centre of Excellence in Analytical Chemistry, University of Sindh, Jamshoro 76080, Pakistan. ${ }^{6}$ Department of Botany, Shah Abdul Latif University, Khairpur 66020, Pakistan. ${ }^{7}$ Department of Chemical Engineering, Faculty of Engineering, University of Selcuk, Campus, 42079 Konya, Turkey.

SN Applied Sciences (2019) 1:144 | https://doi.org/10.1007/s42452-018-0117-5 
with the application of enzyme (glucose oxidase, GOx) for the detection [1, 2]. In this connection many reports have already been published likewise Zhang et al. [3] reported the use of Ag NPs for detection of glucose based on the change in SPR response as a consequence of $\mathrm{H}_{2} \mathrm{O}_{2}$ produced from the enzymatic reaction between glucose and glucose oxidase previously immobilized on surface of $\mathrm{Ag}$ NPs. In a similar scenario, use of gold nanoparticle (Au NPs) coupled with Ag NPs have also been proposed for the detection of glucose [4]. Literature reveals that recently efforts have been carried out for developing fluorescence probe based on GOx loaded nanocomposites of carbon nanodots supported over Ag NPs for gulucose detection. Nanocomplex based on Ag NPs and graphene quantum dots (AgNPs/GQDs) loaded with glucose oxidase have also been used for the development of colorimetric probe for glucose detection [5]. In addition to these, use of bimetallic composite particles and metal oxides has also been reported for the enzyme based optical probes $[6,7]$.

Despite the extreme selectivity offered by enzymatic assays, the use of such probes for practical application is restricted by the limitations offered by the enzyme. The temperature depended stability, high cost and immobilization complexities of the enzyme marks these assays as unfavorable candidates for diverse application. Recently, enzyme-free approaches have gained substantial attention due to their improved sensitivity and selectivity. An optical probe based on nano-mimicking capability of gold and iron oxide magnetic nanoparticles composite has been proposed by Shin et al. [8]. Despite the success of nano-mimicking assays, efforts for finding newer, simpler and cost-efficient protocols for reliable pre-screening of clinically important biomolecules are still under way. In this context, colorimetric sensor which rely on the interaction between the probe (nanoparticle) and target analyte are gaining reasonable consideration [9]. These assays are not only simple in mechanistic consideration but also involve minimum chemicals and complexity which make them suitable to be used practically in low-resource situations [10]. A number of such assays have already been developed for various heavy metal ions and other toxic compounds such as pesticides and drugs $[9,10]$. In the present study we have functionalized Ag NPs with L-cysteine amino acid and carried out direct detection of glucose (i.e. enzyme free) on their surface via colorimetric sensing approach. The assay rely on the competitive interaction between functionalizing agent (L-cysteine) and glucose molecules leading to particle aggregation and subsequent change in SPR response. The developed assay provides a simplest route towards reliable detection of glucose without the need of any enzyme or any additional chemicals. The practical characteristic of the assay has been validated by considering it for glucose detection from human serum samples.

\section{Materials and methods}

\subsection{Chemicals}

All chemicals were analytical grade and used without further purification. Pure Milli-Q water as the preparatory medium. Silver nitrate $\left(\mathrm{AgNO}_{3}-97 \%\right)$, was obtained from E. Merck. L-cysteine $\left(\mathrm{C}_{3} \mathrm{H}_{7} \mathrm{NO}_{2} \mathrm{~S}\right)$, sodium hydroxide $(\mathrm{NaOH}-98 \%)$ and hydrochloric acid $(\mathrm{HCl}-37 \%)$ were purchased from Sigma-Aldrich. The stock solutions of metal salts were prepared in pure Milli-Q water and all experiments were performed at ambient temperature $\left(25 \pm 2^{\circ} \mathrm{C}\right)$.

\subsection{Instrumentation}

UV-Visible spectroscopy (Lambda 35 of PerkinElmer) was considered for monitoring the variation in SPR signal of $\mathrm{Ag}$ NPs within the spectral range of $300-800 \mathrm{~nm}$. Confirmation of surface functionalization was achieved using fourier transform infrared (FTIR) spectroscopy (Nicolet 5700 of Thermo) with ATIR mode. Further, characterizations of functionalized Ag NPs were obtained using transmission electron microscopy (TEM) (Jeol JEM 1200 EX MKI), atomic force microscopy (AFM) and X-ray diffraction (XRD) (model D-8 of Bruker). The change in color was noted by capturing photographs of the colloidal Ag NPs solutions before and after interaction with glucose using 18 mega pixel digital camera.

\subsection{Synthesis and functionalization of silver nanoparticles (Ag NPs)}

The Ag NPs were synthesized and functionalized using one-pot wet-chemical reduction approach. In a typical experiment, a $10 \mathrm{ml} \mathrm{AgNO}_{3}(1 \mathrm{M})$ was homogenized with $1 \mathrm{ml}$ of L-cysteine $(1 \mathrm{mM})$ under constant stirring condition. The clear solution was then introduced into $60 \mathrm{ml}$ of $\mathrm{NaBH}_{4}(1 \mathrm{M})$ until a stable bright yellow colloidal solution was obtained with SPR wavelength of $395 \mathrm{~nm}$. The $\mathrm{pH}$ of formed colloidal solution was maintained at 6.5 in accordance to $\mathrm{pk}_{\mathrm{a}}$ value of L-cysteine to obtain effective functionalization and smaller size. The use of L-cysteine was considered based on its capability to act both as efficient surface protecting agent and mild reducing agent. This allowed preserving homogeneity of synthesized Ag NPs with generation of an inert atmosphere, which controlled the use of protective gas and hence tedious strategies to avoid oxidation. 


\subsection{Colorimetric sensing of glucose using cyst-Ag NPs}

The colorimetric sensing of glucose using cyst-Ag NPs was carried out at room temperature in aqueous solution. For a representative experiment, a certain range of glucose concentration ( $0.05 \mathrm{mM}$ to $1.5 \mathrm{mM}$ ) was introduced within a fixed aliquot of cyst-Ag NPs. The mixtures were allowed to stabilize for few minutes followed by SPR measurement using spectrophotometer. The absorbance was measured at SPR wavelength of $395 \mathrm{~nm}$, in reference to a blank sample (without glucose). The change in absorbance $(\Delta \mathrm{A})$ was taken as the analytical signal for the corresponding concentration, while the change in color from bright yellow to colorless was considered the visual response of the devised sensor. The color change was recorded using an 18 mega pixel digital camera after stabilizing the colloidal mixture for $5 \mathrm{~min}$.

\section{Results and discussion}

\subsection{Characterization of cyst-Ag NPs}

Optical characteristics of nano-sized noble metal particles are a function of geometry and size; thus the optical response of such particles is highly sensitive to change in size of the particles [9]. Owing to the surface plasmon modes of noble metallic NPs which belong to the optical region of electromagnetic spectrum [11-13], one can easily use optical spectroscopy as an efficient tool to observe different molecules based on change in the intra-particle distance of noble metals. In this case, the most stable and small sized cyst-Ag NPs (with blue shift in spectrum) were obtained after careful optimization of reaction parameters like concentrations of the precursor salt and functionalizing agent, $\mathrm{pH}$ of colloidal solution (not shown). Figure 1 shows the UV-Vis absorbance of a represented sample of the as prepared Ag NPs with SPR wavelength noted at $395 \mathrm{~nm}$.

The morphological characteristics were evaluated using TEM analysis. Figure 2 shows a typical image for as-synthesized cyst-Ag NPs. The formed Ag NPs were evident of high surface homogeneity and dispersion indicating the success of surface functionalization with L-cysteine molecules. The average size of cyst-Ag NPs were estimated to be in range of $10-35 \mathrm{~nm} \pm 1.4 \mathrm{~nm}$. The EDX spectra provided as the inset of Fig. 2, further confirms the surface purity of the as-synthesized Ag NPs.

The AFM images for the same Ag NPs are presented in Fig. 3. The findings are apparent of narrow and homogenous distribution which is less likely in case of biological methods where use of plant extracts are considered as reducing/capping agents [14]. The topographical map shows significant variation in topography along the surface. This reflects the smaller cross-section of the synthesized nanoparticles with larger number of active sites available at the surface for reaction participation.

The XRD pattern shown in Fig. 4 consists of peak indexed to (111), (200), (220) and (311) planes of face centered cubic structure (FCC) as referenced against ICCDD (file no: 89-3722). The absence of peaks other than metallic phase silver confirms the compositional purity of the assynthesized cyst-Ag NPs.

Confirmation for the functionalization was obtained using FTIR analysis. Figure 5 shows the FTIR spectra of both standard L-cysteine and Cyst-Ag NPs. The characteristics band for L-cysteine includes bands at 1630 and
Fig. 1 SPR wavelength for cyst-Ag NPs with inset figure showing the corresponding bright yellow colloidal sol

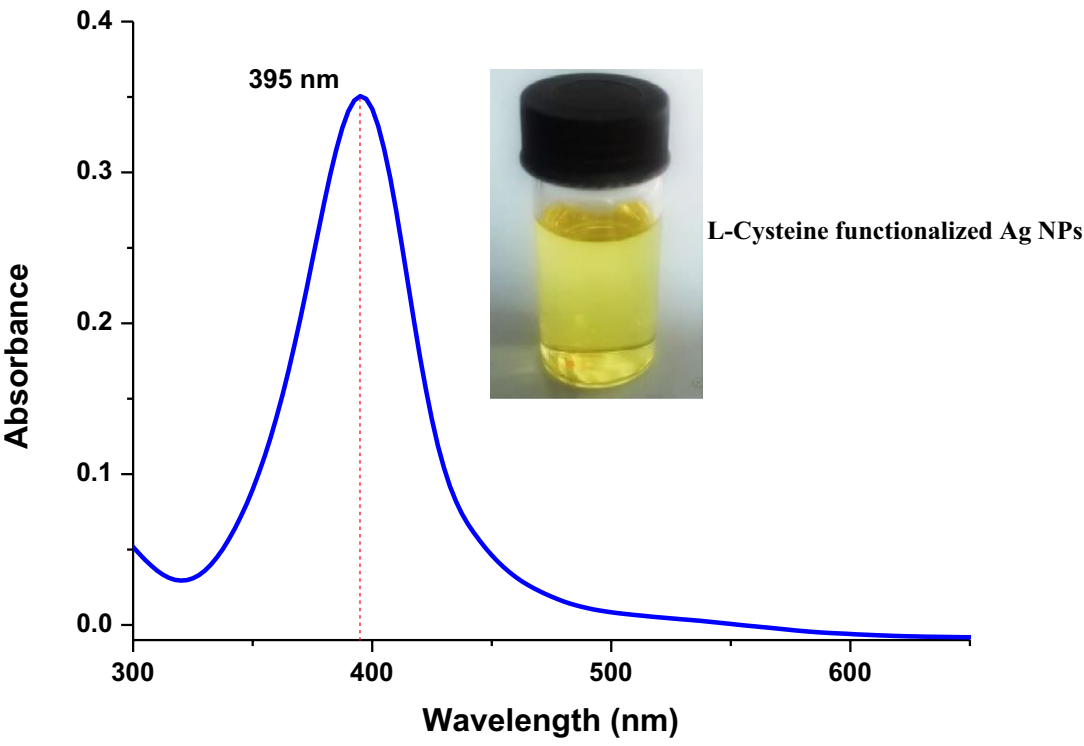

SN Applied Sciences A SPRINGER NATURE journa 

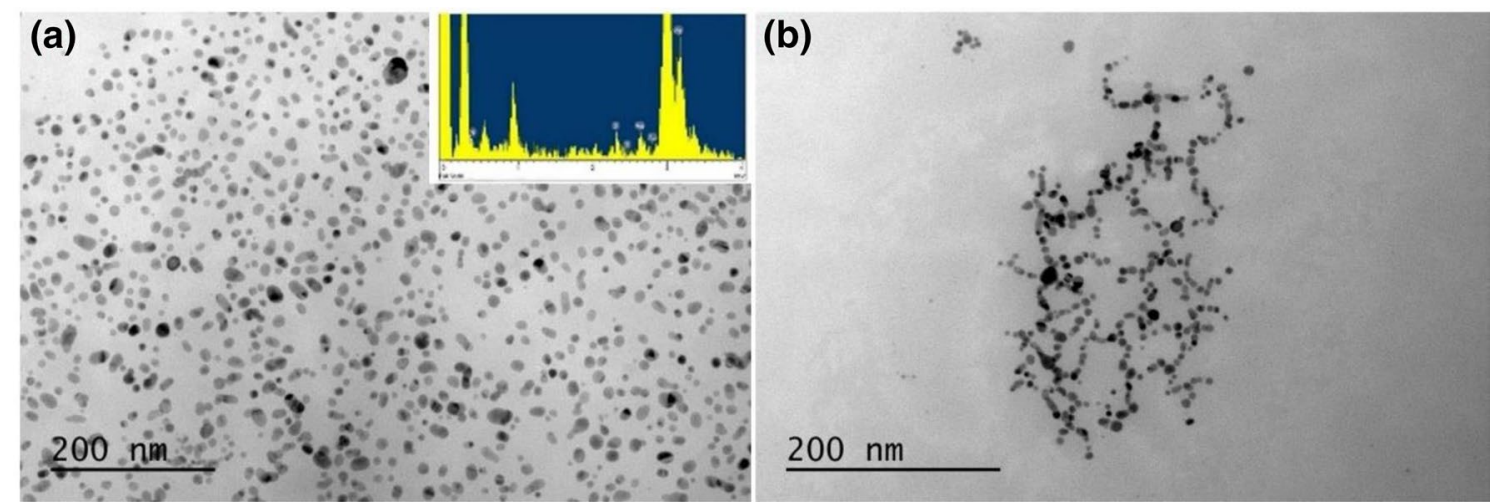

Fig. 2 TEM analysis of a Cyst-Ag NPs with EDX spectra as inset figure and $\mathbf{b}$ Cyst-Ag NPs in the absence and presence of glucose (0.01 $\mu \mathrm{M})$ respectively
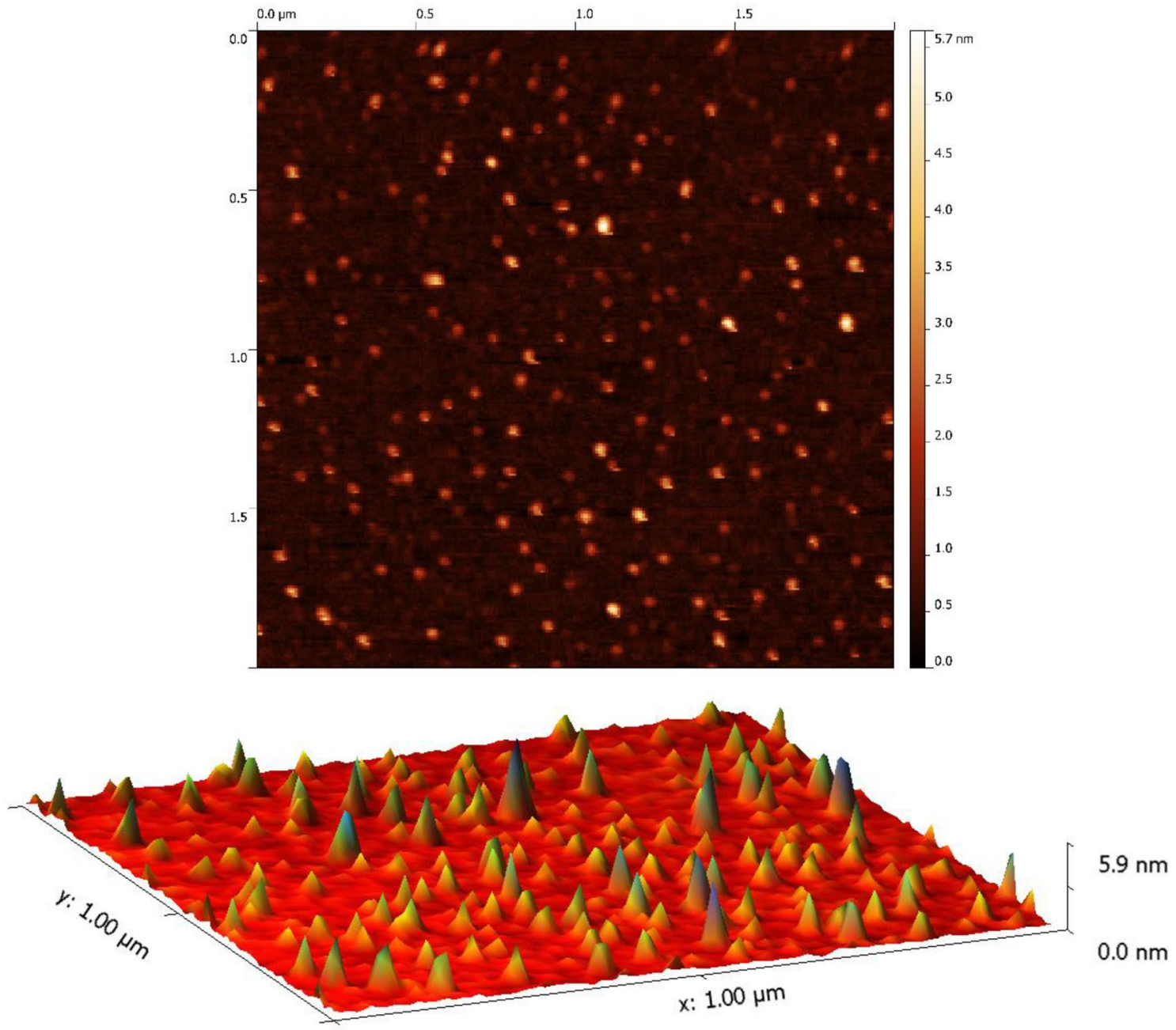

Fig. 3 AFM Height profile with topographical plot indicating the uniform distribution of as-synthesized cyst-Ag NPs 


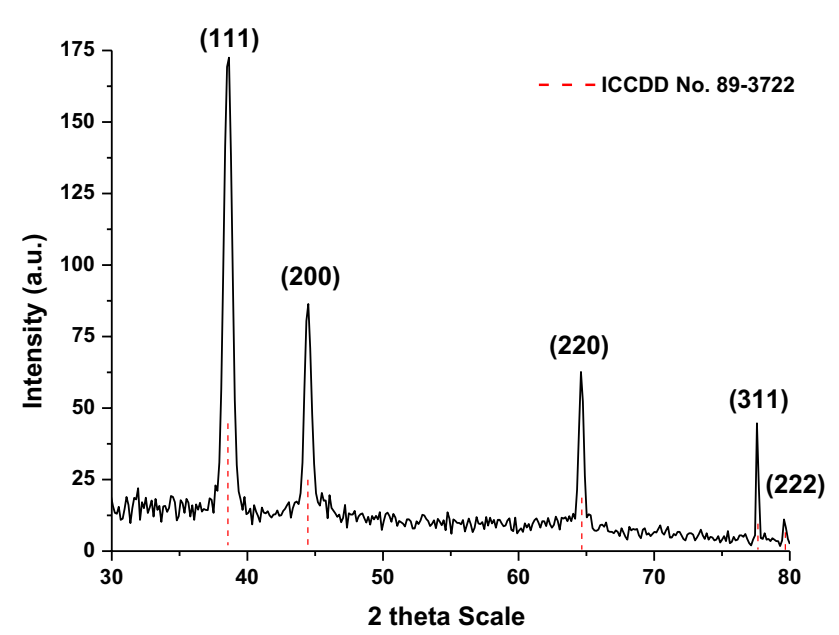

Fig. 4 XRD pattern recorded for Cyst-Ag NPs with major peaks indexed to cubic unit cell of FCC crystal structure for standard silver

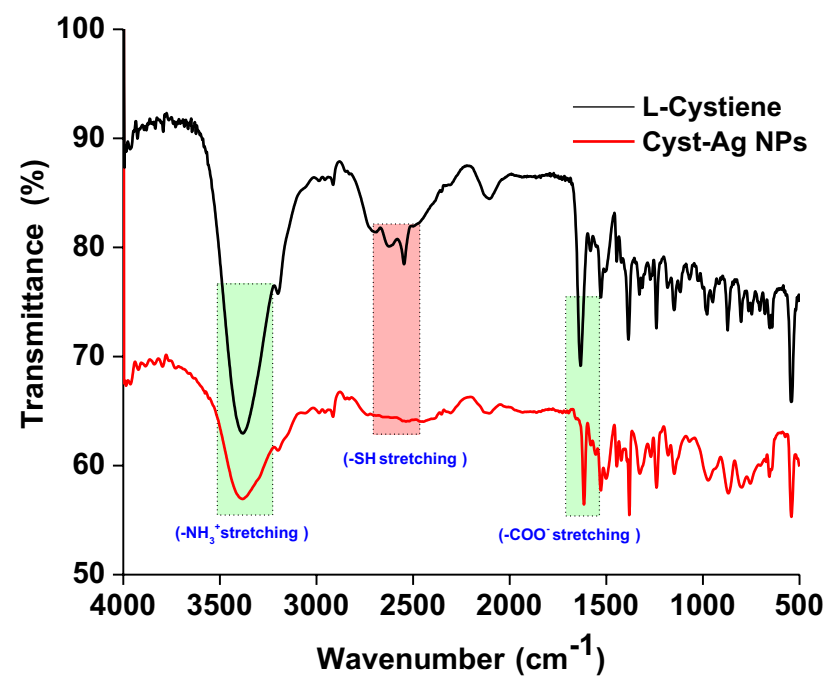

Fig. 5 FTIR profile for standard cysteine and cyst-Ag NPs

$1390 \mathrm{~cm}^{-1}$ for asymmetric and symmetric stretching of $\left(\mathrm{COO}^{-}\right)$and $1530 \mathrm{~cm}^{-1}$ for $-\mathrm{NH}$ bending vibration.

A broad band near 3000 for $-\mathrm{NH}_{3}{ }^{+}$stretching and a band near $2500 \mathrm{~cm}^{-1}$ for $-\mathrm{SH}$ [15-17]. In case of cyst-Ag $\mathrm{NPs}$, the band for -SH was not observed. This confirms the interaction of $\mathrm{Ag}$ with cysteine molecules via thiol group. This interaction was further strengthened by interaction of carbonyl from cysteine over Ag NPs surface, as indicated by shift in the vibrational frequency of $\mathrm{COO}^{-}$from 1630 to $1620 \mathrm{~cm}^{-1}$. A slight shift in the overall frequencies was also observed which can be explained on the bases of dipole change as a result of surface functionalization of Ag NPs with cysteine molecules [18].

\subsection{Enzyme free colorimetric sensing of glucose}

Due to the bright yellow color of colloidal solution of $\mathrm{Ag}$ NPs it exhibit a sharp SPR band near $400 \mathrm{~nm}$ in UV-Vis spectral window. Therefore a typical narrow SPR peak at $395 \mathrm{~nm}$ was evidenced by size homogeneous and crystalline Cyst-Ag NPs as shown in Fig. 1. This noted optical characteristic can be attributed to the effective capping/ functionalizing agent of Ag NPs with cysteine molecules $[19,20]$. The detection mechanism of glucose can be explained on the basis of inter-particle fusion caused by favorable intermolecular forces as a consequence of addition of glucose. This generalized mechanism is illustrated in Scheme 1. It is well-known that SPR of Ag NPs is highly sensitive towards change in the refractive index near the vicinity of metal nanoparticles. In this case when glucose is introduced into colloidal sol of Cyst-Ag NPs, it tends to contribute to the existing inter-ionic forces that prevail between the cysteine functionalized Ag NPs. The glucose based on its hydroxyl functionality can easily facilitates the amine-carbonyl interactions subsequently allowing nanoparticles to fuse together. Unlike the absorptionbased colorimetric sensing where change in inter-particle distance is responsible for the shift in SPR wavelength [21], the said process results in complete aggregation of NPs leading to decline in the SPR intensity. The proposed mechanism is further supported by the TEM analysis of cyst-Ag NPs before and after the introduction of glucose molecules. Figure 3 a shows that in the absence of glucose, the cyst-Ag NPs tends to be spherical and well-dispersed. In contrast, the TEM image Fig. 3b for cyst-Ag NPs with glucose shows evidence of particle fusion and formation of aggregates with size greater than $100 \mathrm{~nm}$. Quantitative response measurements were carried out by monitoring the changes in absorbance $(\triangle A)$ for cyst-Ag NPs with glucose in concentration range of 0.01-0.17 $\mu \mathrm{M}$ (Fig. 6). The linear regression analysis was proof of good linearity with LOD $(3 \times \partial /$ slope $)$ and LOQ $(10 \times \partial /$ slope) estimated to be $1.2 \times 10^{-4}$ and $0.03 \mu \mathrm{M}$ respectively. Table 1 compares the analytical characteristics of the described sensor with other competitor. It is clear that, the discussed sensor offer greater analytical leverage with additional advantage of working without enzyme and cost-friendly. The selectivity of the assay was evaluated by monitoring the change in SPR response against common co-existing interfering species such as ascorbic acid, sucrose and metal ions like $\mathrm{Cu}^{2+}, \mathrm{Zn}^{2+}$ and $\mathrm{K}^{+}$ions with concentration ten folds higher than glucose $(0.01 \mu \mathrm{M})$. The spectral profile shows negligible variation in the SPR intensity of Ag NPs in the presence of interferents (Fig. 7). This negligible variation in the SPR characteristics of Ag NPs, is proof of the high tolerance offered by of cyst-Ag NPs against these interferents. 


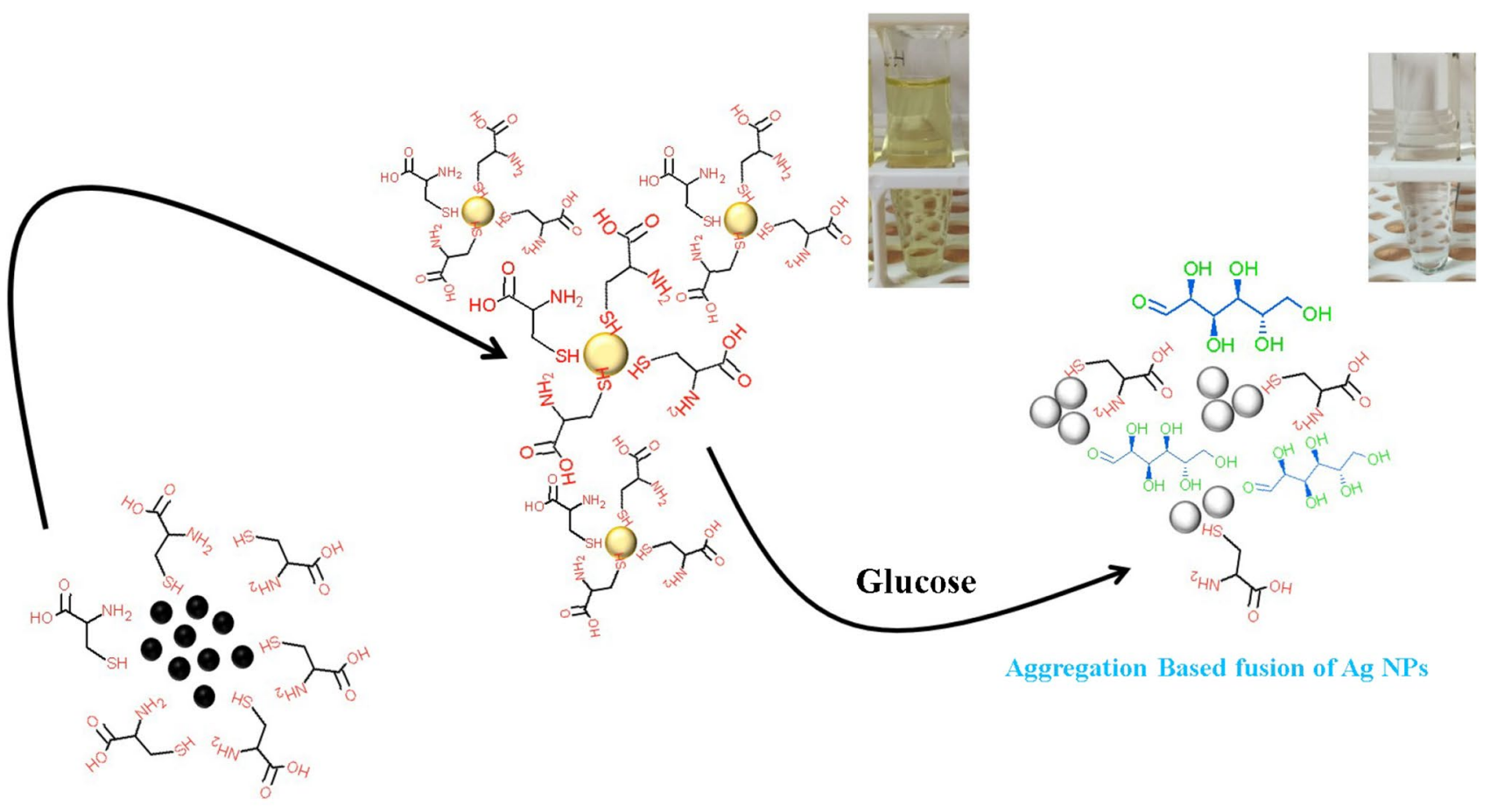

Nucleation in presence of cysteine

Scheme 1 A generalize illustration representing formation and aggregation of Ag NPs in the presence of cysteine and glucose respectively
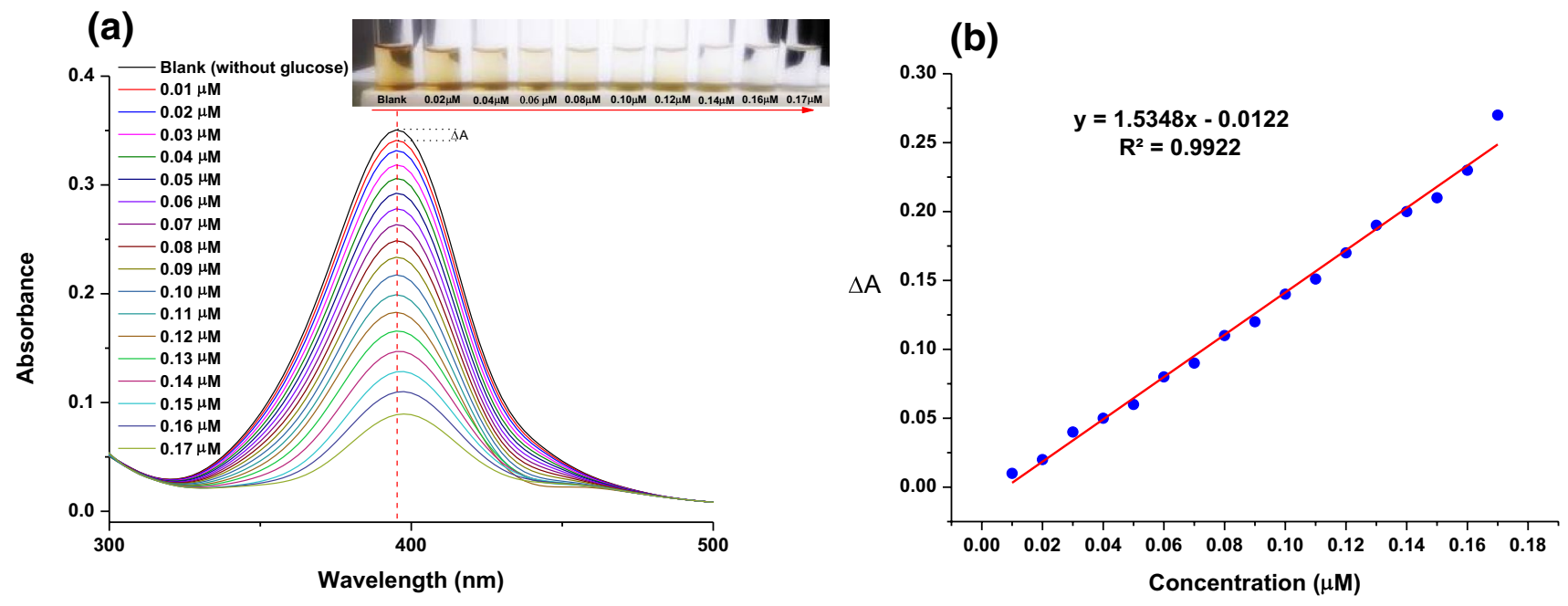

Fig. 6 a Variation in the noted SPR of Cyst-Ag NPs against successive addition of glucose solution with inset figure representing the corresponding decline in the colour intensity and $\mathbf{b}$ Calibration plot

It is clear that, the discussed sensor offer greater analytical leverage with additional advantage of working without enzyme and cost-friendly. The selectivity of the assay was evaluated by monitoring the change in SPR response against common co-existing interfering species such as ascorbic acid, sucrose and metal ions like $\mathrm{Cu}^{2+}, \mathrm{Zn}^{2+}$ and for the devised colorimetric sensor with linear fit analysis for glucose in concentration range of $0.01-0.17 \mu \mathrm{M}$

$\mathrm{K}^{+}$ions with concentration ten folds higher than glucose $(0.01 \mu \mathrm{M})$. The spectral profile shows negligible variation in the SPR intensity of Ag NPs in the presence of interferes (Fig. 7). This negligible variation in the SPR characteristics of Ag NPs, is proof of the high tolerance offered by of CystAg NPs against these interferes. 

of designed sensor with various other competitors
Table 1 Analytical comparison

\begin{tabular}{lllll}
\hline S. no & Type of nanoparticles & Concentrations $(\mu \mathrm{M})$ & LOD $(\mu \mathrm{M})$ & References \\
\hline 1. & Ag Nanoprism & $0.2-100$ & 0.2 & {$[21]$} \\
2. & Ag NPs & 868.6 & - & {$[22]$} \\
3. & Ag NPs & $215.50-3448$ & 99.13 & {$[23]$} \\
4. & Au-Ag NPs & $5-70$ & 3 & {$[24]$} \\
5. & Ag NPs/GQDs & $500-8000$ & 30 & {$[5]$} \\
6. & Isotropic silver nanoparticles & $0.2-32$ & 0.09 & {$[25]$} \\
& (iAg NPs) & $0.5-400$ & & \\
7. & GQDs/Ag NPs & $500-200$ & 0.17 & {$[26]$} \\
8. & Ch-Ag NPs & $2-4000$ & 0.1 & {$[27]$} \\
9. & C Dots/Ag NPs & $0.01-0.17$ & 1.39 & {$[28]$} \\
10. & L-cyst-Ag NPs & & $1 \times 10^{-4}$ & This work \\
\hline
\end{tabular}

Fig. 7 The spectra profile representing the selectivity for cyst-Ag NPs against common interferents with inset figure depicting the corresponding color variation

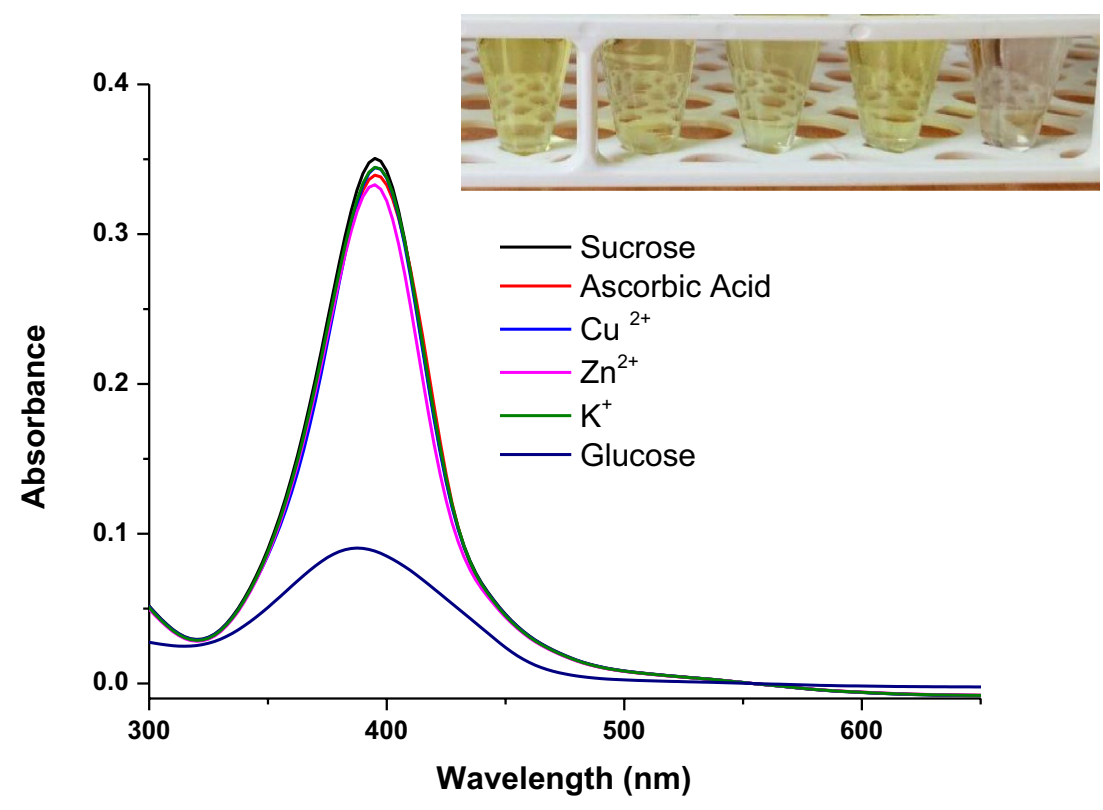

Table 2 Blood glucose determination from real blood serum sample using devised colorimetric probe in reference to measured values obtained using commercial glucometer

\begin{tabular}{llll}
\hline Sample & $\begin{array}{l}\text { Blood glucose } \\
\text { (Glucometer) } \\
\mathrm{mmol} / \mathrm{L}\end{array}$ & $\begin{array}{l}\text { Blood glucose } \\
\text { (Colorimetric sen- } \\
\text { sor) } \mathrm{mmol}^{\mathrm{a}} \mathrm{L}^{\mathrm{a}}\end{array}$ & $\begin{array}{l}\text { Relative standard } \\
\text { deviation (RSD \%) }\end{array}$ \\
\hline 1 & 4.5 & 4.3 & 4.0 \\
2 & 4.8 & 4.4 & 9.0 \\
3 & 5.2 & 5.0 & 4.0 \\
4 & 5.6 & 5.4 & 3.7 \\
\hline
\end{tabular}

${ }^{a}$ The measured concentration is the mean value for three consecutive measurements

\subsection{Testing glucose from human serum samples}

To validate the practical applicability of the developed colorimetric assay, the cyst-Ag NPs were subjected to human serum for glucose analysis taken from lab volunteers with informed consents. Serum was obtained after centrifugation of whole blood followed by two fold dilution for easiness of analysis. The serum samples were then tested for the concentration of glucose according to the procedure mentioned in Sect. 3.2. The concentration measured using Ag based colorimetric assay was cross-checked with the results obtained with commercial glucometer. Table 2 shows the obtained data with RSD value for each samples $<10 \%$ indicating the reliability of the assay for practical applications.

\section{Conclusion}

In conclusion, the study demonstrates the use of L-cysteine functionalized Ag NPs as colorimetric probe for enzyme free detection of blood glucose. The detection system relied on direct interaction between the 
glucose molecules and functional groups of surface bound cysteine molecules. The assay demonstrated excellent working linearity for enzyme free glucose detection in range of $0.01-5.4 \mu \mathrm{M}$. The assay provides a non-enzymatic approach for the detection of glucose avoiding use of toxic chromophores or unstable enzymes like glucose oxidase or HRP. The successful application of the developed sensor in real blood glucose proves its feasibility for practical application and its applicability as detection strips for enzyme free glucose screening method. Moreover, the preparation of this sensor is very simple, fast, inexpensive and extendable to monitor other biologically important molecules.

Acknowledgements We acknowledge The Scientific and Technological Research Council of Turkey (TUBITAK) for provision funding for research expenses, traveling and living expenses to conduct the research during post-doctoral fellowship under 2221-Fellowships for Visiting Scientists and Scientists on Sabbatical Leave.

Funding This study was funded by The Scientific and Technological Research Council of Turkey (TUBITAK) under 2221-Fellowships for Visiting Scientists and Scientists on Sabbatical Leave, the Grant Number of the project is 1059B211600383.

\section{Compliance with ethical standards}

Conflict of interest The authors declare that they have no conflict of interest.

\section{References}

1. Akrema, Rahisuddin (2018) Metal nanoparticles as glucose sensor. In: Khan ZH (ed) Nanomaterials and their applications. Springer, Singapore, pp 143-168

2. Rauf S, Nawaz MAH, Badea M, Marty JL, Hayat A (2016) Nanoengineered biomimetic optical sensors for glucose monitoring in diabetes. Sensors 16(11):1-17

3. Zhang Y, Zhang Y-J, Xia X-D, Hou X-Q, Feng C-T, Wang J-X, Deng $L$ (2013) A quantitative colorimetric assay of $\mathrm{H}_{2} \mathrm{O}_{2}$ and glucose using silver nanoparticles induced by $\mathrm{H}_{2} \mathrm{O}_{2}$ and UV. Chin Chem Lett 24(12):1053-1058

4. Gao Y, Wu YT, Di JW (2017) Colorimetric detection of glucose based on gold nanoparticles coupled with silver nanoparticles. Spectrochimica Acta A Mol Biomol Spectrosc 173:207-212

5. Nguyen ND, Nguyen TV, Chu AD, Tran HV, Tran LT, Huynh CD (2018) A label-free colorimetric sensor based on silver nanoparticles directed to hydrogen peroxide and glucose. Arab J Chem 11(7):1134-1143

6. Zhang X, Wei M, Lv B, Liu Y, Liu X, Wei W (2016) Sensitive colorimetric detection of glucose and cholesterol by using Au@Ag core-shell nanoparticles. RSC Adv 6(41):35001-35007

7. Li Z, Zhang Y, Ye JY, Guo MQ, Chen J, Chen WY (2016) Nonenzymatic glucose biosensors based on silver nanoparticles deposited on $\mathrm{TiO}_{2}$ nanotubes. J Nanotechnol 2016:1-7

8. Shin HY, Cho S, Kim MI (2017) Enzyme-free colorimetric detection of glucose using a composite entrapping gold and magnetic nanoparticles within an agarose gel matrix. J Nanosci Nanotechnol 17(11):7971-7977
9. Soomro RA, Nafady A, Memon N, Sherazi TH, Kalwar NH (2014) L-cysteine protected copper nanoparticles as colorimetric sensor for mercuric ions. Talanta 130:415-422

10. Hussain M, Nafady A, Sirajuddin, Hussain-Sherazi ST, Shah MR, Alsalme A, Kalhoro MS, Mahesar SA, Siddiqui S (2016) Cefuroxime derived copper nanoparticles and their application as a colorimetric sensor for trace level detection of picric acid. RSC Adv 6(86):82882-82889

11. Chen F, Alemu N, Johnston RL (2011) Collective plasmon modes in a compositionally asymmetric nanoparticle dimer. AIP Adv 1(3):032134-16

12. Ghosh SK, Pal T (2007) Interparticle coupling effect on the surface plasmon resonance of gold nanoparticles: from theory to applications. Chem Rev 107(11):4797-4862

13. Sherazi STH, Soomro RA, Uddin S, Memon N (2014) Synthesis and characterizations of highly efficient copper nanoparticles and their use in ultra fast catalytic degradation of organic dyes. Adv Mater Res 829:93-99

14. Ahmed S, Ahmad M, Swami BL, Ikram S (2016) A review on plants extract mediated synthesis of silver nanoparticles for antimicrobial applications: a green expertise. J Adv Res 7(1):17-28

15. Barth A (2007) Infrared spectroscopy of proteins. Biochem Biophys Acta 1767(9):1073-1101

16. Panigrahi S, Kundu S, Basu S, Praharaj S, Jana S, Pande S, Ghosh SK, Pal A, Pal T (2006) Cysteine functionalized copper organosol: synthesis, characterization and catalytic application. Nanotechnology 17(21):5461

17. Sirajuddin, Nafady A, Afridi HI, Sara S, Shah A, Niaz A (2011) Direct synthesis and stabilization of Bi-sized cysteine-derived gold nanoparticles: reduction catalyst for methylene blue. J Iran Chem Soc 8(1):S34-S43

18. Cai Z-X, Yang H, Zhang Y, Yan X-P (2006) Preparation, characterization and evaluation of water-soluble I-cysteine-capped-CdS nanoparticles as fluorescence probe for detection of $\mathrm{Hg}(\mathrm{II})$ in aqueous solution. Anal Chim Acta 559(2):234-239

19. Kalwar NH, Nafady A, Sherazi STH, Soomro RA, Hallam KR, Khaskheli AR, Jamali AA (2015) Catalytic degradation of imidacloprid using L-serine capped nickel nanoparticles. Mater Express 5(2):121-128

20. Kalwar NH, Sirajuddin, Soomro RA, Sherazi STH, Hallam KR, Khaskheli AR (2014) Synthesis and characterization of highly efficient nickel nanocatalysts and their use in degradation of organic dyes. Int J Metals. https://doi.org/10.1155/2014/126103

21. Jazayeri MH, Aghaie T, Avan A, Vatankhah A, Ghaffari MRS (2018) Colorimetric detection based on gold nano particles (GNPs): an easy, fast, inexpensive, low-cost and short time method in detection of analytes (protein, DNA, and ion). Sens Bio-Sensing Res 20:1-8

22. Xia Y, Ye J, Tan K, Wang J, Yang G (2013) Colorimetric visualization of glucose at the submicromole level in serum by a homogenous silver nanoprism-glucose oxidase system. Anal Chem 85(13):6241-6247

23. Serra A, Filippo E, Re M, Palmisano M, Vittori-Antisari M, Buccolieri A, Manno D (2009) Non-functionalized silver nanoparticles for a localized surface plasmon resonance-based glucose sensor. Nanotechnology 20(16):165501

24. Gao Y, Wu Y, Di J (2017) Colorimetric detection of glucose based on gold nanoparticles coupled with silver nanoparticles. Spectrochim Acta Part A Mol Biomol Spectrosc 173:207-212

25. Zong C, Li B, Wang J, Liu X, Zhao W, Zhang Q, Nie X, Yu Y (2018) Visual and colorimetric determination of $\mathrm{H}_{2} \mathrm{O}_{2}$ and glucose based on citrate-promoted $\mathrm{H}_{2} \mathrm{O}_{2}$ sculpturing of silver nanoparticles. Mikrochim Acta 185(3):199

26. Chen S, Hai X, Chen X-W, Wang J-H (2014) In situ growth of silver nanoparticles on graphene quantum dots for 
ultrasensitive colorimetric detection of $\mathrm{H}_{2} \mathrm{O}_{2}$ and Glucose. Anal Chem 86(13):6689-6694

27. Jiang H, Chen Z, Cao H, Huang Y (2012) Peroxidase-like activity of chitosan stabilized silver nanoparticles for visual and colorimetric detection of glucose. Analyst 137(23):5560-5564
28. Ma J-L, Yin B-C, Wu X, Ye B-C (2017) Simple and cost-effective glucose detection based on carbon nanodots supported on silver nanoparticles. Anal Chem 89(2):1323-1328 